\title{
Centros de Recursos Tecnológicos (CRT) en Perú: ¿Redefinen los Recursos Tecnológicos los Procesos de Enseñanza y Aprendizaje?
}

\author{
Carlos David LAURA QUISPE \\ Universidade Federal de Uberlândia (UFU), Minas Gerais, Brasil \\ carlos.quispe@ufu.br \\ Luis Alberto ALMANZA OPE \\ Instituto Superior Tecnológico TECSUP, Arequipa, Perú \\ lalmanza@tecsup.edu.pe
}

\begin{abstract}
RESUMEN. El fenómeno de interés de esta investigación fue la implementación y desarrollo de los Centros de Recursos Tecnológicos (CRT) en Perú, el estudio se desarrolló desde una perspectiva cualitativa bajo las directrices del estudio de casos. La preocupación fundamental del trabajo fue establecer los factores que hicieron insostenible el programa implementado en la educación básica, a través de la deliberación de los hallazgos obtenidos de la interrogante planteada: ¿cuáles son los factores que están relacionados a que los profesores adscritos a los Centros de Recursos Tecnológicos utilicen las tecnologías como herramientas que medien los procesos de enseñanza y aprendizaje? La muestra estuvo conformada por nueve (9) profesores de las tres (3) primeras escuelas que fueron implementadas. Para el levantamiento de la información se utilizó la entrevista semiestructurada, los datos fueron analizados mediante la técnica del análisis de contenido. Los resultados se organizaron en función a las categorías que emergieron del análisis de contenido: 1) diseño e implementación; 2) componente infraestructural; 3) estrategias metodológicas; 4) perfil docente y 5) seguimiento y monitoreo. Este trabajo posibilitó demostrar que la concepción docente predominante siguen siendo las prácticas pedagógicas tradicionales, aún cuando los profesores cuentan con una variada gama de recursos tecnológicos, no fue posible notar el surgimiento de una nueva actitud de los profesores, que conlleve a una educación mediada por la tecnología.
\end{abstract}

Palabras clave: centros de recursos tecnológicos, tecnología en el aula, apropiación tecnológica, evaluación de programas.

\section{Technological Resource Centers (CRT) in Peru: Do Technological Resources Redefine the Teaching and Learning Processes?}

ABSTRACT. The phenomenon of interest of this research was the implementation and development of Technological Resource Centers (CRT) in Peru, the study was developed from a qualitative perspective under the case study guidelines. The main concern of the work was to establish the factors that made the program implemented in basic education unsustainable, through the deliberation of the findings obtained from the question posed: what are the factors that are related to the teachers assigned to the Centers of Technological Resources use technologies as tools that mediate teaching and learning processes? The sample consisted of nine (9) teachers from the three (3) first schools that were implemented. To collect the information, the semi-structured interview was used, the data were analyzed using the content analysis technique. The results were organized according to the categories that emerged from the content analysis: 1) design and implementation; 2) infrastructural component; 3) methodological strategies; 4) teaching profile and 5) monitoring and monitoring. This work made it possible to demonstrate that the predominant teaching conception continues to be the traditional pedagogical practices, even when the teachers have a 
varied range of technological resources, it was not possible to notice the emergence of a new attitude of the teachers, leading to an education mediated by the technology.

Keywords: technology resource centers, classroom technology, technology appropriation, program evaluation.

\section{INTRODUCCIÓN}

La convergencia de las Tecnologías de la Información y la Comunicación (en adelante TIC) ha generado una profunda transformación en nuestra sociedad. Las TIC son parte integral de nuestros lugares de trabajo, escuelas y casas, ellas están cambiando la manera en que vivimos, trabajamos, divertimos, comunicamos, aprendemos y educamos (Furlong \& Davies, 2012; Erstad, 2012; Moyle, 2016). Podemos notar un amplio acceso a estas tecnologías en las escuelas y colegios (Kosma \& McGhee, 2003; Hepp, PÉREZ, Aravena y Zoro, 2017). En este proceso de incorporación de tecnologías a los sistemas educativos, nuestro país no ha sido la excepción. El Perú, así como otros gobiernos latinoamericanos invierten hoy recursos económicos para la incorporación de tecnologías en la educación y, en este sentido, una opción ha sido la implementación de Centros de Recursos Tecnológicos (en adelante CRT).

La incorporación y apropiación de tecnología portátil es un tema vigente en el área de la educación y, ha generado polémica y controversias entre profesores, políticos, investigadores y autoridades educativas. Estudios recientes sobre la temática, como los de: Cuban (2002); Severín \& Capota (2011); Malamud \& Pop-Eleches (2011); Gonzáles y De Pablos (2015); McWillian (2016), indican que no hay evidencias de correlación o asociación entre la tecnología portátil y los procesos de enseñanza y aprendizaje. Malamud \& Pop-Eleches (2011), elaboraron el estudio "Home Computer Use and the Development Human Capital". Los economistas midieron el impacto que tuvo tener computadoras personales, alumnos de clase media baja comparado con el de sus pares que no recibieron computadoras. Encontraron que no hay beneficios y más bien perjuicios una vez que llega la computadora a las escuelas y domicilios. Si bien los alumnos desarrollan las habilidades cognitivas (test de Raven) y computacionales, cae el rendimiento escolar en matemáticas y lectura, aproximadamente en 1/3 de desviación estándar (Malamud \& Pop-Eleches, 2011).

Consistentemente, algunos de los programas implementados en América Latina han resultado decepcionantes tanto en su diseño, su implementación e impacto (Severín \& Capota, 2011). No obstante, algunos estudios señalan que la tecnología pueden mejorar el desempeño de profesores y alumnos, cuando se diseña y planifica una innovación que apunta al fortalecimiento de la función pedagógica de la escuela, otorgando a las computadoras un sentido más allá de sí mismas (Lugo \& Kelly, 2007; Hennessy, 2011).

Por otro lado y, a la luz de lo expresado en los párrafos anteriores, la revisión de la literatura mostró una ausencia de fundamentación teórica para los CRT. En ese sentido, es de vital importancia contar con metodologías que permitan la evaluación sistemática de los CRT, para que sus resultados sirvan de base tanto para el mejoramiento de dichas experiencias, como para la orientación, diseño y planificación de nuevos proyectos. Esta situación motivó el interés por desarrollar un estudio que pudiera investigar los factores que están relacionados a que los profesores adscritos a los CRT no utilicen los recursos TIC en sus prácticas pedagógicas.

La investigación se categoriza como cualitativa y de naturaleza estudio de casos. En este contexto, esta investigación buscó analizar los factores que están relacionados a 
que los profesores adscritos a los CRT utilicen la tecnología como recurso de sus prácticas pedagógicas. En ese sentido, el objetivo de esta investigación fue: identificar los factores que están relacionados a que los profesores adscritos a los CRT empleen los recursos tecnológicos como herramienta de su labor docente. A través de este estudio se busca responder la siguiente interrogante: ¿cuáles son los factores que están relacionados a que los profesores adscritos a los CRT utilicen la tecnología como recurso de su práctica pedagógica? La interrogante se explora principalmente con procedimientos cuantitativos, más específicamente mediante la técnica del Análisis de Contenido (AC).

\section{APROXIMACIÓN CONCEPTUAL}

\subsection{Las Tecnologías de la Información y Comunicación}

Los avances tecnológicos que están produciendo las TIC, llevan a producir cambios radicales en todos los ámbitos del quehacer humano: agilización de los procesos productivos, los medios de comunicación y esparcimiento, el acceso a mayor cantidad de información y otros. Muchos de estos cambios han sido posibles gracias al vertiginoso avance de las TIC de las últimas décadas. Las TIC han permeado todas las organizaciones modernas y son utilizadas hoy en la mayoría de las actividades productivas y de servicios. La tecnología está cambiando radicalmente las formas de trabajo, los medios a través de los cuales las personas se comunican y aprenden, y los mecanismos con que acceden a los servicios que les ofrecen sus comunidades: transporte, comercio, entretenimiento y gradualmente también, la educación, en todos los niveles de edad y profesión, aunque en educación ha tenido mucho menos impacto de lo esperado. (Hinostroza, Labbé y Cerda, 2005; UNESCO, 2016; Grande, Cañon y Cantón, 2016).

\subsection{Las TIC y su Impacto en la Educación}

Ante los constantes cambios y reformas educativas y frente a las crecientes políticas de incorporar TIC en las escuelas, es posible asumir que hay un supuesto de que estos recursos pueden ser un notable aporte en los procesos de enseñanza y aprendizaje, siempre y cuando se tenga en cuenta que las TIC son medios y no fines, son herramientas y materiales de construcción que facilitan el aprendizaje, el desarrollo de habilidades y distintas formas de aprender (Sánchez, 2000; Oliver \& Iñiguez, 2017). Existe un creciente interés en el rol que las TIC pueden desempeñar para mejorar la educación y los colegios. Kosma, postula que ciertas características de las nuevas tecnologías son consistentes con los principios de las ciencias del aprendizaje y que prometen mejorar la educación (Kosma \& McGhee, 2003). Las TIC se constituyen en la actualidad como poderosos recursos educativos que el profesor puede utilizar en sus prácticas pedagógicas con miras a mejorar la calidad de las mismas (Hepp, 1999; Slechtova, 2015). No obstante, a decir de Bonilla "las TIC no fueron concebidas para la educación; no aparecen naturalmente en los sistemas de enseñanza; no son "demandadas" por la comunidad docente; no se adaptan fácilmente a usos pedagógicos" (Bonilla, 2003: 120).

\subsection{TIC y Profesores}

La implementación de nuevos entornos de aprendizaje depende, fundamentalmente de las acciones del docente, Pero esas acciones, para ser efectivas, deben ser acompañadas de una mayor autonomía para tomar decisiones, modificar sus actitudes, desarrollar propuestas de trabajo en equipo y usar TIC (Valente, 1999; Venn \& Vraking, 2009). Lo que implica, desafiar sistemas de creencias y rutinas institucionalizadas de prácticas 
pedagógicas. Por lo tanto, en términos de integración de TIC, esto puede requerir una reformulación de las nociones básicas de la cultura de las instituciones educativas respecto de lo que constituye el contenido y sus aplicaciones, lo que comprende el aprendizaje y el tiempo comprometido; el cambio en la escuela debe involucrar a todos los participantes del proceso educativo. Alumnos, profesores, directores, especialistas y comunidad en general (Fullan, 2002; Venn \& Vraking, 2009).

Los profesores son los actores directos cuando, de apropiación tecnológica en el aula se trata. Como señala Zhao y otros, el profesor es naturalmente la primera persona que puede observar los factores que afectan el uso de la tecnología en el aula. Son por lo tanto, los principales observadores, ejecutores y evaluadores, lo cual no quiere decir que sean los que realicen estas tareas de la mejor manera (Zhao et al., 2001). De este modo, los profesores han sido los principales implicados en la incorporación de TIC en las escuelas, sin embargo existen preocupaciones de que las expectativas de uso poco exitosas no erosionen la motivación y predisposición que tienen los profesores (Venn \& Vraking, 2009; Macia y Garreta, 2018).

\subsection{Centros de Recursos Tecnológicos (CRT)}

\subsection{1 ¿Desde dónde y por qué surgen los CRT?}

Entre los años 2007 y 2012, y como anticipo de lo que podría ser un solvente proceso de incorporación e integración de tecnologías a la educación peruana, el gobierno ha venido ensayando un conjunto de disposiciones encaminadas a reglamentar y fortalecer algunas dimensiones decisionales en relación a la implementación de programas de incorporación de tecnologías a la educación peruana. Los Centros de Recursos Tecnológicos se crean mediante la Resolución Ministerial $\mathrm{N}^{\circ}$ 0339-2009-ED, esta asumió el formato de "Normas para la creación y desarrollo de los Centros de Recursos Tecnológicos (CRT)” (MINEDU, 2008, p. 5)

Los CRT, son un escenario donde se organiza los recursos TIC para su aplicación en ambientes como: el aula de innovación pedagógica, el aula de clases, la biblioteca y otros espacios no convencionales, como salas de lectura o espacios abiertos de la Institución Educativa. Un CRT, cuenta con los siguientes recursos tecnológicos: 1) laptop XO, 2) Un servidor con capacidad de 500 gigas más un estabilizador, 3) un acceso de Point de WIFI (Router), 4) una laptop convencional para uso del docente en el CRT, 5) USB con portal educativo, 6) panel solar, 7) estabilizador, 8) un proyector multimedia, 9) un KIT de robótica por cada 4 estudiantes que ocupan el CRT, 10) laptop para docente, 11) ecram, 12) estación VSAT, 13) textos en castellano, quechua y aymara y, 14) guías para el docente en castellano (INEI, 2011, MINEDU, 2012).

\section{METODOLOGÍA}

Para realizar la presente investigación se optó por el estudio de casos fundamentado principalmente según las propuestas de Yin (2001), Gil (2010) y Stake (1999). Técnica que se caracteriza por buscar una representatividad de hacia conceptos que iluminen el conocimiento sobre algún fenómeno en particular (Yin, 2001). De esta forma, también, se hace más eficiente la muestra, pues no es necesario llegar a un gran número de escuelas para alcanzar lo que se conoce como el punto de saturación de los estudios, que es el punto en donde los elementos encontrados se repiten consistentemente, es decir los nuevos informantes ya no proporcionan información nueva y relevante (Glaser \& Strauss, 1967).

\subsection{Sujetos Informantes}


Se realizó un muestreo teórico, los sujetos informantes fueron profesores que participaron de la capacitación en el uso de los CRT. Se identificaron profesores que puedan brindar la mayor información posible sobre el proceso de incorporación y apropiación de los CRT en sus prácticas pedagógicas. Los criterios de selección fueron los siguientes: a) profesores que estén dispuestos a ser entrevistados; b) profesores que realicen innovaciones con los CRT; y c) profesores de distinto género y edad. El número de docentes entrevistados fueron nueve (9), las edades de los docentes fluctuaban entre los 37 y 52 años; en cuanto al género, 3 fueron hombres y 6 fueron mujeres; en cuanto al régimen laboral, 3 de ellos eran contratados y 6 eran nombrados.

\subsection{Técnicas de recolección de datos}

\subsubsection{Entrevista Semi estructurada}

Se eligió trabajar con entrevistas, pues ellas son consideradas como una de las "principales técnicas de trabajo en casi todos los tipos de pesquisa utilizados en las ciencias sociales" (André \& Ludke, 1986, p. 33). La entrevista puede definirse como un proceso en el que interactúa el entrevistador y el entrevistado para obtener información que sea útil a la investigación, lo común es que una entrevista se parezca mucho más a una conversación que a una instancia formal con respuestas previamente categorizadas (Marshall \& Rossman, 1995; Ramírez, 2002; Ortiz, 2007). La entrevista es utilizada para recoger datos descriptivos en el leguaje del propio sujeto, permitiendo al investigador desarrollar intuitivamente una idea sobre la manera como los sujetos interpretan diferentes aspectos del mundo (André \& Ludke, 1986).

\subsection{Procedimientos para el análisis}

Una vez recogida toda la información a través de las entrevistas semi estructuradas. En seguida, se buscó analizar la información, a través del análisis de contenido de Bardin (1977), que en sus palabras es: "Un conjunto de técnicas de análisis de las comunicaciones buscando obtener, por procedimientos, sistemáticos y objetivos de descripción del contenido de los mensajes, indicadores que permitan la inferencia de conocimientos relativos a las condiciones de producción/recepción (variables inferidas) de estos mensajes" (Bardin, 1977, p. 42).

\subsection{Aspectos Éticos}

Se tuvo en cuenta solicitar las autorizaciones necesarias para el ingreso a las escuelas, se comunicó en forma clara y precisa los objetivos de la investigación. Los sujetos informantes tienen pleno derecho a conocer cómo será utilizada la información que ellos proporcionan a los investigadores (Belmont, 1979; Di Conza y Quiroga, 2013). Con el propósito de proteger el anonimato de los informantes solo se les identificó por su edad y género, por ejemplo (profesor hombre, 37 años).

\section{RESULTADOS Y DISCUSIÓN.}

Las entrevistas fueron transcritas y analizadas a través del análisis de contenido (Bardin, 1977). Esta metodología de análisis fue elegida principalmente por que se caracteriza como una metodología que produce y expresa sentidos, permitiendo que el investigador alcance otras formas de interpretación sobre lo que está investigando, a partir de sus conocimientos empíricos y teóricos. A partir de las entrevistas transcritas, producimos 98 unidades, que contenían en sí significados importantes para el propósito de nuestra investigación. Las unidades pasaron por un proceso de categorización inicial, en el cual se determinó 47 categorías; seguidamente se procedió a realizar una categorización 
intermedia, de donde emergieron 18 categorías, finalmente, emergieron 5 categoría finales: 1) diseño e implementación; 2) componente infraestructural; 3) estrategias metodológicas; 4) perfil docente y 5) seguimiento y monitoreo.

\section{1 diseño e implementación}

En relación a esta categoría, se pudo constatar que los CRT en Perú adolecen, en su ejecución, de la existencia de una política explícita para la implementación y desarrollo del programa, de problemas administrativos y logísticos. El programa carece de un detallado diagnóstico, inexistencia de metas claras. Si bien se pretendió que la educación básica peruana marchara de acuerdo con las nuevas tendencias mundiales, la motivación primordial al parecer fueron los intereses políticos de corto plazo. Esta interpretación se basa en las siguiente declaración: "En general, los recursos tienen que compartirse porque no nos dotaron del número suficiente de computadoras y recursos en general, al parecer no hubo planificación, entonces muchas veces pasa por el criterio de quienes dirigen, de quien administra la educación, y hay que decirlo nunca se pensó en nosotros, nunca se pensó en los niños" [SIC] [Profesor hombre, 39 años].

A partir de lo manifestado por los maestros en el párrafo anterior, los CRT en Perú, no han sido capaces de irse adaptando a las necesidades y exigencias impuestas por los docentes, las diferentes realidades y, la comunidad educativa en general. Se puede afirmar que el principal problema fue ponerlo en marcha sin haber realizado un exhaustivo y apropiado diagnóstico. Por otra parte, la incorporación de la tecnología ha tenido lugar de la mano de una serie de promesas, publicidad y amplias expectativas en cuanto a los logros que esta traería para la educación. Promesas que aún no han sido satisfechas suficientemente (Venzky, 2004; Warschauer \& Ames, 2010).

Las innovaciones tecnológicas tiende a desajustar las estructuras organizativas de la escuela: distribución de espacios, horarios, formación de grupos, funciones y tiempos de los profesores para trabajo colegiado, etc. Este nuevo contexto de prácticas pedagógicas con incorporación de tecnologías, ha modificado el estado actual de las prácticas pedagógicas tradicionales, a partir de las herramientas que se incorporan en el aula. En su gran mayoría los maestros no se sienten capacitados para llevar adelante sus prácticas pedagógicas haciendo uso de los recursos tecnológicos, como se puede percibir en el siguiente fragmento: "me siento un poco desconcertada, porque estamos dentro de un programa para el cual no estamos capacitados, solamente tenemos una orden de ejecutar algo, pero no estamos capacitados debidamente y bueno tenemos que tratar de hacer lo posible cada día con los niños " [SIC] [Profesora mujer, 40 años].

Lo descrito en párrafos anteriores también se evidencia en los planteamiento como los de Siqueira quien sostiene que no basta informatizar la escuela, incorporando computadoras, componentes electrónicos, herramientas tecnológicas, recursos TIC, pues junto a ello se necesita de capacitación, entrenamiento y preparación de los profesionales que irán a interactuar con los recursos tecnológicos (Siqueira, 2004).

\subsection{Componente Infraestructural}

En lo que respecta a infraestructura tecnológica, las deficientes condiciones de infraestructura, es uno de los problemas más significativos, se debe destacar las características deterioradas de las computadoras y los problemas técnicos permanentes. Lo anterior puede corroborarse en la siguiente afirmación: "no me siento cómoda, como le dije muchas de las computadoras han empezado a fallar, yo no tengo conocimientos técnicos, entonces si una computadora falla, solo la separo. También 
las actividades que trae la computadora no están de acuerdo con la comunidad, no están de acuerdo con mi manera de enseñar" [SIC] [Profesor hombre, 45 años].

Otro elemento que define el bajo nivel de uso de los recursos TIC es la mala calidad y mantenimiento inadecuado del hardware, también es importante dar cuenta sobre la falta de contenidos y herramientas digitales, prácticamente la totalidad de los docentes señala que no cuentan con recursos de calidad y, que los existentes en las computadoras son ajenos al contexto de las escuelas, al respecto el siguiente informante señala: "No le doy importancia, claro que tampoco me opongo, pero tiene sus límites y eso conlleva a casi no hacer nada. Pero si hablamos, primero que nada que nos proporcionen una buena computadora, bien implementada, con todo lo que tiene que tiene que ser, con todo lo que se requiere, no como las que nos ha mandado el gobierno que en sí, sólo es entretenimiento para los niños; [...] en general yo sólo uso la computadora para operaciones básicas: sumas, restas y división no más. $Y$ a veces para uno que otro juego" [SIC] [profesor hombre, 51 años].

\subsection{Estrategias metodológicas}

La siguiente dimensión de análisis refiere principalmente a aspectos relacionados con la metodología, los CRT, implican cambios en las actitudes de los maestros que permitan innovar en las prácticas pedagógicas. Para que los profesores hagan uso total de las tecnologías en sus trabajo es necesario que hagan cambios radicales en la manera o forma de cómo enseñan (Macia y Garreta, 2018). Respecto a la metodología se abordan aspectos como la falta de preparación en estrategias metodológicas con el uso de los recursos TIC, falta de políticas claras para insertar los recursos TI, por ejemplo uno de los docentes informa: "Debieron venir especialistas para que nos capaciten, sobre todo en nuevas estrategias de aprendizaje, por que sin duda al usar estas computadoras, nuestra metodología también tiene que cambiar, más no fue así, como le dije, sólo nos entregaron un documento" [SIC] [profesor hombre, 46 años]

Los profesores también hacen una autocrítica a su labor pedagógica, como se puede notar en la siguiente declaración: "[...] Aún seguimos con el método tradicional de dictado, es muy difícil cambiar de la noche a la mañana, me parece que como complemento de nuestra actividad docente dentro del aula es positivo, aunque tiene sus limitaciones [...] las actividades de las computadoras no están de acuerdo a nuestro contexto, es como si nos quisieran imponer ciertas cosas, ciertos modelos" [SIC] [profesor hombre, 45 años].

La introducción de las nuevas tecnología demandan nuevos e importantes papeles a los profesores, un punto fundamental es que los docentes deberían redefinir sus roles tradicionales; resultaría indispensable mostrarles las maneras de utilizar las nuevas herramientas en forma eficaz (Zhao et al., 2001). Entonces, no es la simple incorporación de tecnologías a la educación que irá cambiándola, pues el profesor puede seguir utilizando la tecnología sólo para motivar viejas prácticas pedagógicas. Al incorporar las tecnologías en la educación es necesario pensar el prácticas que posibiliten las relaciones horizontales y la construcción colectiva de conocimientos entre profesores y alumnos (Kensky, 2003). Los aplicativos virtuales puede generar condiciones favorables para el aprendizaje significativo, pues no son lineales ni dependen del tiempo (Ellwanger et al., 2014).

Esta situación se empeora cuando indagamos sobre la relación tecnología/alumno, si bien en un primer momento hubo un gran interés del alumno por manipular las computadoras, con el paso del tiempo los niños y niñas muestran una absoluta 
indiferencia frente a las computadoras: "[...] Si bien la tecnología llegó como una novedad, esto con el paso del tiempo ha dejado de ser así, pues los niños poco a poco van perdiendo el interés por trabajar con ellas, más que todo debido a que se han ido deteriorando con mucha facilidad" [SIC] [profesor hombre, 51 años].

Las tecnologías exigen que los docentes desempeñen nuevas funciones y también, requieren nuevas metodologías. Estos cambios obligan a romper con las prácticas tradicionales en las cuales prevalece la clase frontal y el concepto del profesor como un ente rígido. Lograr la integración de las tecnologías en el aula dependerá de la capacidad de los maestros para estructurar el ambiente de aprendizaje de forma no tradicional, fusionar las tecnologías con nuevas pedagogías y fomentar clases dinámicas en el plano social, estimulando la interacción cooperativa, el aprendizaje colaborativo y el trabajo en grupo (UNESCO, 2008).

\subsection{Perfil docente}

El siguiente foco de análisis se identificaron aspectos relacionados tales como: creencias negativas de los maestros, rechazo de la tecnología por parte de los profesores, aspecto generacional y subutilización de los recursos tecnológicos. Con la llegada de la tecnología a las aulas, se da inicio a una serie de creencias negativas en la mayoría de los profesores, los maestros perciben que su ambiente de trabajo ha sido modificado, de allí que tenga una actitud de desconfianza.

El principal problema de los docentes de la generación digital, es que la sociedad actual cambia muy rápidamente. Los profesores se han formado y se están formando con una cultura y una visión del significado de sus profesión que ya ha cambiado (Gros \& Silva, 2005). Las ideas anteriores se relacionan con lo citado por la siguiente docente: "Un poco desconcertada, es que nosotros no nacimos con esto, y los niños sí nacieron con esto, para los niños sí ha sido muy significativo el cambio, es que ellos no miden las consecuencias, ellos por ensayo y error descubren las cosas muy rápido, nosotros pensamos en las consecuencias que nos puede traer tal o cual acción, se puede echar a perder si presiono esto. Y esto es lo que muchas veces me desconcierta, pues para poder llevar acabo mi labor pedagógica, yo tengo que liderarla, de lo contrario, impera el desorden, la indisciplina" [SIC] [profesora mujer, 40 años].

Otro informante sostiene que los niños tienen más facilidad para manipular la tecnología: "[...] Los niños han descubierto mucho más rápido que yo todo lo que contiene la computadora con el sólo hecho de manipularla en un día" [SIC] [profesor hombre, 49 años]. Para finalizar esta categoría es necesario recordar lo que sostiene Quaresma et al. (2014), es muy importante la influencia del conocimiento previo sobre el uso de la tecnología y, el manejo y dominio tecnológico del profesor para la apropiación de la tecnología.

\subsection{Seguimiento y monitoreo}

En su mayoría los profesores señalan que no existe ningún tipo de seguimiento y monitoreo por parte de funcionarios del Ministerio, siempre aparecen expresiones como "se olvidaron de nosotros", "nos dejaron a nuestra suerte", "a nadie le interesa", llaman la atención por ejemplo las siguiente declaración: "Este programa es sólo puras historias. Es como que nos dieron un juguete nuevo, al principio tratamos de usarla, pero luego te vas dando cuenta que el aporte que nos brindan estos recursos es casi nulo, entonces como que paulatinamente hemos dejado de usarlos y hemos vuelto a nuestra manera de trabajar. Que al final me parece que es más productivo que trabajar 
con tecnología. Además, a estas alturas ya a nadie le interesa, ya ni vienen los del ministerio" [SIC] [profesor hombre, 46 años].

La incorporación de la tecnología en las escuelas van de la mano con la capacidad que tengan los directivos ministeriales, para desarrollar un adecuado seguimiento y evaluación, así también políticas explícitas que por un lado permita focalizar las prioridades de los maestros y por otro lado, implementar estrategias de reparación y reposición de las computadoras averiadas. En las siguientes líneas puede notarse la preocupación de los profesores en relación a estos aspectos: "Yo creo que falta un poco más de seguimiento, de repente así como usted está ahora preguntándome sobre las dificultades, sobre los aciertos, bueno en un primer momento si no, vinieron los primeros meses pero de ahí ya no se ha visto un poco la ausencia de ellos, parece que de repente deberían hacer el seguimiento, escuchar nuestras opiniones y de acuerdo a eso también superar las deficiencias que se nos presentan a los maestros sobre todo rurales" [SIC] [profesora mujer, 42 años]. Algunos desafíos pueden ser previstos y encarados durante la etapa de planificación, mientras que otros surgen durante la implementación y desarrollo. El monitoreo continuo ayuda a identificar obstáculos en una etapa temprana (Sucker \& Bonifaz, 2005).

\section{REFLEXIONES FINALES.}

El diseño, implementación y desarrollo de este programa, llamado "Centros de Recursos Tecnológicos", no ha estado ajeno a dificultades propias de una innovación que genera grandes cambios en un espacio que ha sido muy estable: la sala de clases. Los resultados de nuestro estudio, demuestran por un lado, que la mayoría de los profesores que componen la muestra estratégica analizada, tienen una imagen de disconformidad sobre el efecto esperado de la innovación por el programa CRT; por otro lado, se hacen explícito un conjunto de elementos que impiden que los docentes hagan uso efectivo de los recursos TIC.

Orientados por la pregunta de investigación: ¿cuáles son los factores que están relacionados a que los profesores adscritos a los CRT utilicen la tecnología como recurso de su práctica pedagógica? Este estudio ha permitido establecer los principales aspectos que impiden que los profesores hagan uso adecuado de los diversos recursos TIC. De esta manera y coincidiendo con lo que reporta la literatura especializada. En concreto, el análisis se realiza en función a las cinco categorías emergentes: 1) diseño e implementación; 2) infraestructura tecnológica; 3) estrategias metodológicas; 4) perfil docente y 5) seguimiento y evaluación.

En segundo lugar, un aspecto a considerar es la infraestructura tecnológica, las deficientes condiciones de infraestructura, es una de las limitaciones más significativas; así como la ausencia de materiales y recursos TIC de acuerdo a los diferentes contextos de trabajo. Es preciso destacar las características deterioradas de las computadoras y recursos TIC. Según los informantes muchas de las computadoras e implementos ya habrían cumplido su ciclo de vida, sin embargo hasta la fecha no existe un plan de reposición.

En tercer lugar, los CRT no han previsto una estrategia que cambie las prácticas pedagógicas de los maestros y maestras en las aulas, sólo ha propuesto un cambio en cuanto al número y cantidad de computadoras, equipos y recursos en las escuelas. Según lo indicado en el marco teórico y en el desarrollo de la investigación para el éxito de este tipo de innovaciones en la educación es fundamental; establecer modelos pedagógicos pertinentes de uso e integración de las computadoras, es evidente que los 
docentes no logran compartir modalidades de trabajo con las computadoras, esto se relaciona con el nivel de dominio que tienen de las mismas. Los profesores no han modificado la estructura ni el método pedagógico del desarrollo de sus prácticas pedagógicas por la incorporación de las computadoras. En general, los profesores tienden a confiar más en lo que tradicionalmente les ha funcionado, por lo cual no están abiertos a innovaciones que involucren computadoras portátiles.

En cuanto al perfil docente, con la llegada de las tecnologías al aula, se da inicio a una serie de creencias negativas sobre estos recursos. En los discursos de los profesores manifiestan que su ambiente de trabajo ha sido perturbado, que las computadoras en lugar de ayudar su labor la dificulta aún más; en su mayoría consideran a las computadoras como una carga más; de allí que tengan una actitud de rechazo o desconfianza sobre su aplicabilidad en el aula, ellos pueden carecer del conocimiento de cómo las computadoras pueden apoyar su carrera profesional. Esto retarda el proceso de apropiación de la computadora, pues le resta autonomía y seguridad profesional. La adopción tecnológica es una decisión personal, no influenciado por otra gente y la presencia de recursos o limitada por el colegio.

Más aún, los sistemas de seguimiento y evaluación, de acuerdo con nuestras entrevistas, los CRT no cuentan con un apropiado seguimiento y evaluación; desde instancias del Ministerio de Educación no valoran las posibilidades de interacción y comunicación con los principales actores del programa, limitando sus instancias para mejorar el programa; encontrando un obstaculizador para nutrir y mejorar el programa a partir de sus experiencias. Se percibe entonces una falta de seguimiento y coordinación entre el Ministerio de Educación y los profesores. Probablemente, lo que el Ministerio de Educación considere pertinente y necesario, no vaya de la mano con lo que los maestros realmente requieren y necesitan.

AGRADECIMIENTO: al Consorcio de Investigación Económica y Social (CIES), de Perú; a la Universidade Federal de Uberlândia (UFU), de Minas Gerais, Brasil; a la Universidad Católica Santa María (UCSM), de Perú.

\section{REFERENCIAS BIBLIOGRÁFICAS}

ANDRÉ, M.; LUDKE, M. Pesquisa em Educação: abordagems qualitativas. São Paulo: E. P. U. 1986.

BARDIN, L. Análise de conteúdo. Lisboa: Edições 70. 1977.

BELMONT R. Informe Belmont. Principios éticos y normas para el desarrollo de las investigaciones que involucran a seres humanos. PDR. Reports. 1979.

BONILLA, J. Políticas nacionales de educación y nuevas tecnologías: el caso de Uruguay. Educación y Nuevas Tecnologías. Experiencias en América Latina. IIPE. UNESCO, Buenos Aires. 2003.

CUBAN, L. Oversold and Underused: Computers Classroom. Boston: Hardvard University. 2002.

DI CONZA, J., QUIROGA, C. Las responsabilidades éticas en la publicación de artículos científicos. Revista Argentina de Microbiología. 45(4): 219-221. 2013.

ELLWANGER, A.; ROSSATO, J.; BORTOLUZZI, V.; FAGAN, S. Objetos de aprendizagem no ensino de tópicos de eletrodinâmica. RENOTE. CINTED-UFRGS. Vol. 12, No 1. 2014.

ERSTAD, O. The learning lives of digital youth. Beyong formal and informal. Oxford Reviw of Educaction. 38(1), pp. 25-43. 2012. 
FULLAN, M. Leading and learning for the 21stC. Vol $1 \mathrm{~N}^{\mathrm{o}}$ 3-January 2002. The latest ideas on school. 2002.

FURLONG, J.; DAVIES, C. Young people, new technologies and learning at home: taking context seriously. Oxford Review of Education, 38(1), 45-62. 2012.

GIL, A. Como elaborar projetos de pesquisa. 5 ed. São Paulo: Atlas. 2010.

GLASER, B.; STRAUSS, A. The Discovery of Grounded Theory: Slralegiesi for Qualitative Research, Chicago, Aldine. 1967.

GONZÁLES, A.; DE PABLOS, J. Factores que dificultan la Integración de las TIC en las Aulas. Revista de Investigación Educativa, 33(2), 401-417. 2015.

GRANDE, M.; CAÑON, R.; CANTÓN, I. Tecnologías de la Información y la Comunicación: Evolución del Concepto y Características. International Journal of Educational Research and Innovation (IJERI). Vol. 6. pp. 218-230. 2016.

GROS, B.; SILVA, J. La formación del profesorado como docentes en los espacios virtuales de aprendizaje. En Revista Iberoamericana de Educación. N ${ }^{\circ}$ 36. 2005. Disponible en: http://www.campus-oei.org/revista/tec_edu32.htm Acceso en: 02 de abril de 2017.

HENNESSY, S.The role of digital artefacts on the interactive whiteboard in supporting classroom dialogue. Journal of Computer Assisted Learning, 27, 463-489. 2011.

HEPP, P. Enlaces: Todo un mundo para niños y jóvenes de Chile. En García Huidobro, J. E. (Ed.), La reforma educacional chilena, Editorial Popular, Madrid. 1999.

HEPP, P.; PÉREZ, M.; ARAVENA, F.; ZORO, B. Desafíos para la integración de las TIC en las escuelas: Implicaciones para el liderazgo educativo. Pontificia Universidad Católica de Valparaíso. Valparaíso, Chile. 2017.

HINOSTROZA, E.; LABBÉ, C.; CERDA, C. Modelo pedagógico para la integración de las tecnologías al currículo y manual de prácticas pedagógicas. Instituto de informática educativa. Universidad de la Frontera. 2005.

INEI (2011). Glosario de términos educativos. Encuesta Nacional a Instituciones Educativas de Nivel Inicial y Primaria. ENEDU, 2011. Dirección Nacional de Censos y Encuestas. Lima. Perú. 2011.

KENSKY, V. Tecnologias e ensino presencial e à distancia. Campinas. SP: Papirus. 2003.

KOZMA, R., y MCGHEE, R. ICT and innovative classroom practices. In R.B. kozma (Ed.), Technology, Innovation and Educational Change (pp. 43-80). Eugene: International Society for Technology in Education. 2003.

LUGO, M.; KELLY, V. La gestión de las TIC en las escuelas: el desafío de gestionar la innovación. Buenos Aires: IIPE-UNESCO. 2007.

MACÍA, M.; GARRETA, J. Accesibilidad y Alfabetización Digital: Barreras para la Integración de las TIC en la Comunicación Familia y Escuela. Revista de Investigación Educativa, 36(1), 239-257. 2018.

MALAMUD, O.; POP-ELECHES, C. Home Computer Use and the Development Human Capital. Quarterly Journal of Economics. Vol. 126, № 2: pp. 987-1027. 2011.

MARSHALL, C.; ROSSMAN, G. Designing Qualitative Research. $2^{\circ}$ Edition. SAGE Publications, USA. 1995.

MCWILliAN, E. Shifting centres: Pedagogical relations in the Era of Big Data, International Journal of Leadership in Education, 19(1), 40-55. 2016.

MINEDU. Manual del docente para el uso de la laptop XO. Dirección General de Tecnologías Educativas. Lima. 2008.

MINEDU. Balance del programa Una Laptop por Niño. Dirección General de Tecnologías Educativas. Lima. Perú. 2012. 
MOYLE, K. Leadership and learning with ICT. Voices from the profession. Australian Institute for Teaching and School Leadership. 2016.

OLIVER, M. INIGUEZ, L. The use of information and communication technologies (ICTs) in health centers: the practititoners' point of view in Catalonia, Spain. Interface (Botucatu). 21(63):945-55. 2017.

ORTIZ, F. La entrevista de investigación en las ciencias sociales. México: Limusa. 2007.

QUARESMA, C.; ABEGG, I.; GARCÊS, S.; FELIX, R. Tecnologias na educação: inclusão digital dos professores da rede estadual a partir da implementação do programa tablet educacional. RENOTE. CINTED UFRGS. Vol. 12; N: 1. Porto Alegre, 2014.

RAMÍREZ, L. El Estudio de Caso y sus herramientas de investigación aplicada. Université de Sherbrooke, Faculté d'administration. Sherbrooke. 2002.

SÁNCHEZ, J. Aprendizaje visible, tecnología invisible. Santiago: Dolmen Ediciones. Universidad de Chile. 2000.

SÁNCHEZ, J. (2003). Integración curricular de TIC concepto y modelos. Departamento de ciencias de la computación. Universidad de Chile. Santiago. 2003.

SEVERÍn, E.; CAPOTA, C. Modelos Uno a Uno en América Latina y el Caribe. Panorama y perspectivas. Banco Interamericano de Desarrollo (BID). División de Educación. 2011.

SLECHTOVA, P. Las actitudes de los estudiantes de pregrado a la utilización de las TIC en la Educación. Procedia - Social and Behavioral Sciences 171 (2015) pp. 1128 1134. 2015.

STAKE, R. Investigación con estudio de casos. $2^{\text {a }}$ ed. Ediciones Morata. S. L. Madrid. 1999.

SIQUEIRA, E. Como viviremos. Sao Paolo. Brasil: Ed. Saraiva. 2015.

UNESCO. El Futuro de la educación en América Latina y el Caribe. Santiago de Chile. UNESCO. 2008.

UNESCO. Competencias y estándares TIC desde la dimensión pedagógica: Una perspectiva desde los niveles de apropiación de las TIC en la práctica educativa docente. Pontificia Universidad Javeriana, Cali Colombia. 2016.

VALENTE, J. O Computador Na Sociedade do Conhocimento. NIED. CAMPINAS. São Paulo. 1999.

VENN, W.; VRAKKING, B. Homo zapiens: educando na era digital. Tradução Vinicius Figueira. Porto Alegre: Artmed. 2009.

VENEZKY, R. Technology in the Classroom: steps toward a new vision. Education, Communication \& Information, Vol. 4, No 1 Taylor \& Francis Group. 2004.

WARSCHAUER, M.; AMES, M. ¿Can One Laptop per Child Save the World's Poor?. Journal of International Affairs, Fall/Winter, Vol. 64. N ${ }^{\circ} 1.2010$.

YIN, R. Estudo de caso: planejamento e métodos. Trad. Daniel Grassi. 2. ed. Porto Alegre: Bookman. 2001.

ZHAO, Y., PUGH, K., SHELDON, S., BYERS, J. Conditions for Classroom Technology Innovations. Teachers College Record. Vol. $104 \mathrm{~N}^{\circ}$ 3, pp. 482-515. 2001. ZUCKER, A.; BONIFAZ, A. Lessons Learned About Providing Laptops For All Students. NEIR-TEC. 2005 